DOI: $10.21892 / 978-958-5547-34-6.4$

\title{
PRÁCTICAS PARENTALES, SINTOMATOLOGÍA ANSIOSA Y RENDIMIENTO ACADÉMICO EN ESTUDIANTES ADOLESCENTES, SAN PEDRO - SUCRE
}

\author{
Paula Vanessa Acosta Castilla ${ }^{1}$ \\ Fundación Mujer Siglo XX1 \\ Alexandra María Cáez Quesada² \\ Yulieth Patricia Dávila Serpa ${ }^{3}$ \\ Enlace de Primera Infancia, Alcaldía Municipal de Buenavista Sucre
}

\section{Resumen}

El estudio tiene como propósito determinar si existe relación entre las prácticas parentales, la sintomatología ansiosa y el rendimiento académico de los estudiantes de secundaria de la institución educativa San Juan Bosco de San Pedro, Sucre. Son pocas las investigaciones realizadas considerando las citadas variables, ello indica que este es un estudio innovador a nivel departamental, razón que lo justifica. En relación con la metodología, la investigación es descriptiva, transeccional y su diseño no experimental, de corte correlacional. Para llevarlo a cabo se tomó una muestra estratificada de 166 estudiantes, 9 estudiantes del grado sexto, 25 del grado séptimo, 32 del grado octavo, 37 del grado noveno, 35 del grado decimo y 28 del grado undécimo, con edades comprendidas entre los doce y dieciocho años. Como instrumentos se utilizó la escala de prácticas parentales y el cuestionario de ansiedad estado/rasgo (STAI); a partir de los resultados arrojados se pudo constatar que el bajo rendimiento académico es influenciado por la forma negligente de los cuidadores en la crianza de sus hijos, del mismo modo, se pudo establecer

1 Psicóloga. Correo: Paula.acosta@cecar.edu.co.

2 Psicóloga. Correo: alexandra.caez@cecar.edu.co.

3 Psicóloga. Correo: yulieth.davila@cecar.edu.co. 
que existe una relación entre la ansiedad estado y el estilo parental autoritario y democrático.

Palabras clave: rendimiento académico, prácticas parentales, sintomatología ansiosa, adolescentes.

\section{Abstract}

The purpose of the study is to determine whether there is a relationship between parental practices, anxious symptoms and the high school students' academic performance of the educational institution San Juan Bosco, in San Pedro, Sucre. There are few investigations published that consider the aforementioned variables, this indicates that this is an innovative study at a departmental level, which justifies it. In relation to the methodology, the research is descriptive, transactional, non-experimental and correlational. To carry it out, a stratified sample of 166 students was taken, 9 students of the sixth grade, 25 of the seventh grade, 32 of the eighth grade, 37 of the ninth grade, 35 of the tenth grade and 28 of the eleventh grade, aged between twelve and eighteen. As instruments, the parental practices scale and the state/trait anxiety questionnaire (STAI) were used. From the results obtained, it was found that the low academic performance is influenced by the negligent of their caregivers in the upbringing, in the same way, it could be established that there is a relationship between the anxiety and authoritarian and democratic parental style.

Keywords: academic performance, parental practices, anxious symptomatology. 


\section{Introducción}

El presente estudio se centró en determinar la relación entre rendimiento académico, sintomatología ansiosa y prácticas parentales. Implicó una revisión de la concepción teórica de estos aspectos; en tal sentido, se puede señalar que el rendimiento académico es definido por Tonconi (2010) como el nivel demostrado de conocimientos en un área, evidenciados en indicadores cuantitativos, usualmente expresados mediante calificación ponderada en el sistema vigesimal.

Respecto a la variable ansiedad: esta constituye una respuesta anticipatoria a una amenaza futura -de acuerdo con la definición proporcionada por el manual diagnóstico y estadístico de los trastornos mentales-. Es importante recalcar que hemos considerado los postulados de Baumrind (1966), quien define las prácticas parentales como las acciones de los padres dirigidas a cumplir con los objetivos de los niños, al mismo tiempo, también constituye una forma de mantener las normas al igual que el respeto por la autoridad.

Considerando esta revisión teórica preliminar, surge la inquietud de conocer si los factores determinantes en el proceso académico de los estudiantes están ligados al núcleo familiar y a la conducta de los mismos. Se tomó como referencia la Institución Educativa San Juan Bosco de San Pedro, Sucre, la cual no está exenta de las dificultades que aquejan a nuestro sistema educativo. Desde esta perspectiva, es importante señalar el informe realizado por la Organización para la Cooperación y el Desarrollo Económico (OCDE, 2016) el cual expone que mediante la realización de la prueba PISA entre los países de Latinoamérica, Colombia, a nivel regional, obtuvo uno de los puntajes más bajos en las tres áreas evaluadas (Programa para la Evaluación Internacional de Alumnos, 2012). Por consiguiente, se encuentra dentro de los 10 países que tiene mayor número de estudiantes con bajo rendimiento académico; evidenciando así las múltiples falencias del sistema educativo colombiano, sumado a esto, la falta de cooperación entre las diferentes instituciones que sostienen a la sociedad actual del país, resaltando en primera instancia la familia.

En los últimos años el rendimiento académico en el país se ha visto bastante cuestionado debido a que los resultados obtenidos no han sido los mejores, esta problemática se ha visto ligada a otra inquietud, un 
cuestionamiento que surge a partir de lo anteriormente mencionado y es: ¿Qué tan comprometidos están los padres en el proceso educativo de sus hijos? O por el contrario, ¿qué tanta presión ejercen sobre este? Espitia y Montes (2009) han señalado que la familia es la primera Institución Educativa, su dinámica media el aprendizaje y desarrollo de sus miembros. Por lo que se hace necesario que los cuidadores participen de forma activa en el proceso educativo de los estudiantes, pues resulta complejo que las instituciones logren una adecuada labor por sí solas, resaltando así que la educación es un trabajo integral entre la institución y el núcleo familiar.

A través del informe realizado por PISA - 2015 se dio a conocer que dependiendo del contexto sociodemográfico donde habitan los estudiantes, varían sus resultados educativos (OCDE, 2016). Por otra parte, otro factor que involucra las vertientes de estudio envuelven los estilos parentales, en este sentido, según los postulados de Baumrind (1966) existen diferentes tipos de crianzas o practicas parentales que forjan el proceso de crecimiento y formación de las personas, algunos pueden llegar a ser bastante flexibles mientras que otros son muy demandantes, creando algunas veces emociones, pensamientos, así como conductas contraproducentes de quienes viven bajo dichos términos.

Lipps et al. (2012) señala que los padres son los seres que más influyen en la vida de cualquier persona, siendo esta influencia profunda y perdurable, por tal razón los estilos de crianza que los padres implementan en sus hijos están determinados por las vivencias favorables o desfavorables que ellos tuvieron durante su formación por parte de sus cuidadores. Atendiendo a esta consideración, las prácticas parentales son las estrategias que los padres emplean como reglas o pautas de enseñanzas para sus hijos, estas se emplean de acuerdo a la educación que hayan recibido los padres y el contexto en el que crecieron, en muchos casos trascienden generaciones, implantando esquemas mentales, los cuales pueden contribuir de manera positiva o negativa en el ámbito escolar.

Sobre la base de las ideas expuestas se hace necesario estudiar la sintomatología ansiosa, la cual es un problema de salud mental que afecta, a quien lo padece, en diferentes ámbitos de la vida. En el caso de los estudiantes, se podría ver afectado, más específicamente, en su rendimiento académico, dependiendo del contexto sociocultural que influye en el estudiante. Según estudios realizados por Ospina, Hinestrosa, Paredes, 
Guzmán y Granados (2011) uno de cada dos niños y adolescentes en Colombia - y en el mundo- padecen un tipo de sintomatología afectiva, originando poca funcionalidad en la esfera social y familiar de la persona, ello es muy alarmante, pues deteriora su desarrollo afectivo y calidad de vida.

Indagaciones realizadas con los directivos de la Institución Educativa San Juan Bosco de San Pedro - Sucre, revelan que se han estado presentando diferentes problemáticas con estudiantes de dicha Institución, manifestándose desde bajo rendimiento académico, conductas agresivas y discusiones entre compañeros y con profesores. Otra problemática de trasfondo denota que muchos de estos estudiantes conviven en sus hogares con graves dificultades: desde mala o nula comunicación con sus padres, hasta casos de violencia física y psicológica; de esta situación derivó la necesidad de estudiar las practicas parentales que están siendo implementadas en los estudiantes e indagar cómo se relacionan con su conducta (sintomatología ansiosa) y su rendimiento académico.

Las ideas precedentes permiten contextualizar este estudio, llevado a cabo en el municipio de San Pedro, Sucre, específicamente en la Institución Educativa San Juan Bosco, ubicada en el barrio Kennedy, tomando como población estudiantes adolescentes. Dicha institución cuenta con 17 salones de clases en buen estado para la preparación y la formación de los estudiantes en su proceso de aprendizaje. Asimismo, entre los espacios de estudios que posee, cuenta con: biblioteca, aula virtual o sala de investigación; herramientas tecnológicas necesarias para el proceso de aprendizaje. Para la cantidad de estudiantes que tiene la institución, es un lugar muy pequeño, requiriendo espacios de recreación, zonas verdes o zonas de esparcimientos que contribuyan al buen comportamiento de los estudiantes. Con respecto a su matrícula de estudiantes, tiene una cantidad menor a la de las otras Instituciones Educativas públicas del municipio.

Es importante profundizar la problemática vigente con los estudiantes, realizar estudios que permitan analizar su comportamiento y la forma en que estos puedan verse afectados, desmejorando su rendimiento académico a causa de factores internos y externos. La mayoría de las investigaciones encontradas a nivel nacional e internacional, se han desarrollado con estudiantes universitarios y no con estudiantes en bachillerato. Particularmente en Sucre, son pocas las investigaciones 
realizadas en instituciones educativas, considerando variables como la ansiedad, las prácticas parentales y el rendimiento académico, lo cual indica que este constituye un estudio innovador a nivel departamental, razón que lo justifica, pues se espera dar respuestas a las diferentes problemáticas que se vivencian hoy en día en las instituciones del país, aportando posibles soluciones para el mejoramiento del desempeño académico, la calidad de vida de los estudiantes y la calidad de educación de la institución.

En esta investigación, para determinar la relación entre las prácticas parentales, la sintomatología ansiosa y el rendimiento académico de los adolescentes, se formularon los siguientes objetivos específicos: analizar las prácticas parentales; determinar la presencia de sintomatología ansiosa; identificar el rendimiento académico y establecer la relación entre prácticas parentales, sintomatología ansiosa y rendimiento académico en estudiantes de secundaria de la Institución Educativa San Juan Bosco de San Pedro Sucre; destacando así, que es de gran importancia saber que en el desempeño académico de los estudiantes influyen diversas variables que, de alguna manera, pueden favorecer o afectar al estudiante en su formación.

\section{Metodología}

La metodología engloba un conjunto de aspectos que orientan sobre la finalidad del estudio y las estrategias para la recolección de la información o los datos. En este sentido, la presente investigación es de tipo descriptivo, pues permite caracterizar un hecho, fenómeno, o sujetos, para indicar su estructura o comportamiento, sin influir en el mismo, es decir, no hay manipulación de los hechos o variables, estos criterios responden a los planteamientos de Hernández, Fernández y Baptista (2010).

En relación al diseño, no experimental, transversal, correlacional; no se realiza una manipulación deliberada de variables, su propósito es describirlas y analizar su incidencia e interrelación en un momento dado. De este modo, la presente indagación tiene como propósito establecer si existe relación entre las prácticas parentales, la sintomatología ansiosa y el rendimiento académico de los adolescentes, sin manipular las variables. 


\section{Procedimiento}

Para llevar a cabo el proceso de recolección de datos, en principio, nos dirigimos a la Institución Educativa San Juan Bosco, ubicada en el municipio de San Pedro, Sucre, con el propósito de hacer entrega al coordinador de dicha Institución una la carta de presentación donde se explicó la finalidad de la investigación. Posteriormente, se entregó un consentimiento informado a los estudiantes pertenecientes a la muestra, para que sus cuidadores dieran la autorización de participar en el estudio. Para seguir con la aplicación de la Escala de prácticas de crianza de los hijos (PPS) fue necesario hacer una aplicación pilotaje de la escala a 60 estudiantes adolescentes en edades comprendidas entre 12 y 18 años, de la institución Educativa Altos del Rosario, del municipio de Sincelejo, Sucre; con el fin de contextualizar el instrumento a la población colombiana, ya que este test no ha sido validado en el país, pero ha sido utilizado en otras investigaciones anteriormente. Finalmente, se aplicó la Escala de prácticas de crianza de los hijos (PPS) y el Cuestionario de autoevaluación ansiedad estado/rasgo -STAI a 166 estudiantes de la Institución Educativa San Juan Bosco.

\section{Población y muestra}

La población del estudio está conformada por 294 estudiantes adolescentes de la Institución Educativa San Juan Bosco de San Pedro Sucre, de la cual se seleccionó una muestra estratificada de 166 estudiantes distribuidos de la siguiente manera, 9 estudiantes del grado sexto, 25 del grado séptimo, 32 del grado octavo, 37 del grado noveno, 35 del grado decimo y 28 del grado undécimo con edades comprendidas entre los 12 y 18 años.

\section{Instrumentos}

Se aplicaron dos instrumentos, el primero de estos es el Cuestionario de Autoevaluación Ansiedad Estado/Rasgo - STAI (Spielberger, Gorsuch y Lushene): está conformado por 40 ítems, de los cuales 20 evalúan la ansiedad estado (A/E), y 20 ansiedad rasgo (A/R), ambos anotados en una escala de 4 puntos. La aplicación del STAI comprende una duración de aproximadamente 15 minutos, puede ser aplicado a adolescentes y adultos 
-con niveles de cultura mínimo para comprender las instrucciones y enunciados del cuestionario-

El Cuestionario STAI está compuesto por dos escalas separadas de autoevaluación que miden dos conceptos independientes de la ansiedad: (a) la ansiedad estado (A/E), se describe como una condición emocional transitoria del organismo humano, caracterizada por sentimientos subjetivos de tensión y aprensión, así como hiperactividad del Sistema Nervioso Autónomo; puede variar con el tiempo y fluctuar en intensidad; (b) la Ansiedad rasgo (A/R) señala una relativamente estable propensión ansiosa por la que difieren los sujetos en su tendencia a percibir las situaciones como amenazadoras y a elevar, consecuentemente, su ansiedad estado (A/E).

El segundo instrumento es la Escala de prácticas de crianza de los hijos (PPS): el propósito de esta es evaluar las estrategias que los padres o cuidadores utilizan para cuidar e interactuar con sus hijos. Está constituida por 29 ítems anotados en una serie de 5 puntos, donde 1 es nunca, 2 raramente, 3 a veces, 4 con frecuencia y 5 muy frecuente. PPS puede ser aplicada a adolescentes, con una duración recomendada de aproximadamente 15 minutos.

\section{Análisis estadístico}

Para llevar a cabo esta investigación, se efectuó la tabulación de los resultados en Microsoft Excel, posteriormente se hizo necesario el uso del Paquete Estadístico Para las Ciencias Sociales SPSS, en el cual se hizo una correlación entre las variables de estudio.

Con respecto al análisis de los resultados de este estudio se utilizaron tablas de frecuencia, medidas de tendencia central, como la media y desviación estándar, del mismo modo se utilizó el coeficiente de correlación de PEARSON, que es una medida paramétrica estadística.

\section{Resultados}

Los resultados se presentan divididos en aparatados, en coherencia con los objetivos de la investigación, estos se han dispuesto en el siguiente orden: caracterización demográfica de la muestra poblacional; 
prácticas parentales en estudiantes adolecentes; sintomatología ansiosa en estudiantes adolecentes; rendimiento académico; y por último, las prácticas parentales, sintomatología ansiosa y rendimiento académico en estudiantes adolecentes.

\section{Caracterización demográfica de la muestra poblacional}

Dentro de la muestra poblacional hubo mayor presencia de adolescentes correspondiente al sexo masculino, que al sexo femenino, la Tabla 1 (Datos demográficos de la población) muestra con más detalle la información sobre edad y escolaridad de los participantes.

\section{Tabla 1}

Datos demográficos de la población

\begin{tabular}{cccc}
\hline \multicolumn{2}{c}{ Variables } & N & $\%$ \\
\hline \multirow{2}{*}{ Sexo } & Chicas & 80 & 48,2 \\
& Chicos & 86 & 51,8 \\
\hline \multirow{4}{*}{ Edad } & 12 & 8 & 4,8 \\
& 13 & 36 & 21,7 \\
& 14 & 29 & 17,5 \\
& 15 & 33 & 19,9 \\
& 16 & 27 & 16,3 \\
& 17 & 20 & 12,0 \\
& 18 & 13 & 7,8 \\
\hline \multirow{4}{*}{ Escolaridad } & $6^{\circ}$ & 9 & 5,4 \\
& $7^{\circ}$ & 25 & 15,1 \\
& $8^{\circ}$ & 32 & 19,3 \\
& $9^{\circ}$ & 37 & 22,3 \\
& $10^{\circ}$ & 35 & 21,1 \\
& $11^{\circ}$ & 28 & 16,9 \\
\hline
\end{tabular}

Fuente: elaboración propia.

\section{Prácticas parentales en estudiantes adolecentes}

De acuerdo con los resultados obtenidos el estilo de prácticas parentales que más prevalece en la muestra poblacional es el democrático (con 39,2\%), seguido del estilo negligente, autoritario y por último permisivo, como se puede evidenciar en la siguiente tabla. 
Tabla 2

Prácticas parentales

\begin{tabular}{lcc}
\hline Tipo de práctica parental & N & $\%$ \\
\hline Práctica parental autoritaria & 33 & 19,9 \\
\hline Práctica parental democrático & 65 & 39,2 \\
\hline Práctica parental permisiva & 17 & 10,2 \\
\hline Práctica parental negligente & 51 & 30,7 \\
\hline
\end{tabular}

Fuente: elaboración propia.

\section{Sintomatología ansiosa en estudiantes adolecentes}

Una vez obtenida la información a través del cuestionario STAI, se observa un gran porcentaje de población con sintomatología ansiosa, tal como se especifica en la Tabla 3.

Tabla 3

Sintomatología ansiosa

\begin{tabular}{lccccc}
\hline & N & Mínimo & Máximo & Media & $\begin{array}{c}\text { Desviación } \\
\text { estándar }\end{array}$ \\
\hline $\begin{array}{l}\text { P. Directa - Ansiedad } \\
\text { Estado }\end{array}$ & 166 & 0 & 45 & 15,40 & 8,203 \\
$\begin{array}{l}\text { P. Directa - Ansiedad } \\
\text { Rasgo }\end{array}$ & 166 & 4 & 52 & 20,44 & 9,081 \\
\hline
\end{tabular}

Fuente: elaboración propia.

\section{Rendimiento académico}

A continuación, se muestra en la Tabla 4 el acumulado de notas en relación al rendimiento académico, obtenido a través de los dos primeros periodos correspondientes al año 2017.

Tabla 4

Rendimiento académico

\begin{tabular}{cccccc}
\hline & N & Mínimo & Máximo & Media & $\begin{array}{c}\text { Desviación } \\
\text { estándar }\end{array}$ \\
\hline Notas & 166 & 1,99 & 4,40 & 3,31 & 3,2 \\
\hline
\end{tabular}

Fuente: elaboración propia. 


\section{Prácticas parentales, sintomatología ansiosa y rendimiento académico en estudiantes adolecentes}

Para la interpretación de los datos es importante tener en cuenta la correlación de Pearson, la cual establece que cuando el índice de los datos arrojados se encuentra más cerca a cero (0) existe mayor correlación entre variables, mientras que si el índice se acerca más a uno (1) la correlación es menor. Si la correlación es positiva es mayor para ambas variables, pero si es negativa la correlación, es mayor una variable y menor la otra.

Haciendo referencia a la correlación entre variables se infiere que la ansiedad estado y la ansiedad rasgo correlacionaron negativamente con la parentalidad autoritaria, esto quiere decir que, a menor estilo parental autoritario mayor ansiedad estado y mayor ansiedad rasgo, lo cual significa que en este estudio los niños con ansiedad no tienen padres autoritarios.

La ansiedad estado se correlaciona positivamente con el estilo parental democrático; es decir, a mayor práctica democrática, mayor ansiedad estado. Esto resalta que en las familias con estilo democrático los hijos pueden presentar mayor sintomatología ansiosa, considerando a su vez, que el estilo democrático puede tener influencia de manera funcional en la ansiedad estado. Del mismo modo, el estilo parental democrático se correlaciona de forma negativa con la ansiedad rasgo, lo cual indica que, a mayor estilo democrático implementado por los padres, menor ansiedad rasgo presentan los hijos.

De igual forma, dentro de los resultados se obtuvo que las notas guardan una línea de relación con la parentalidad autoritaria, esto significa que a mayor estilo parental autoritario, los estudiantes obtienen mejores notas, pero son menos ansiosos.

La ansiedad estado correlacionó con el estilo parental autoritario y democrático. Las notas se relacionan con el estilo parental negligente: son más bajas con un alto nivel de negligencia. 
Prácticas parentales, sintomatología ansiosa y rendimiento académico en estudiantes adolescentes, San Pedro - Sucre

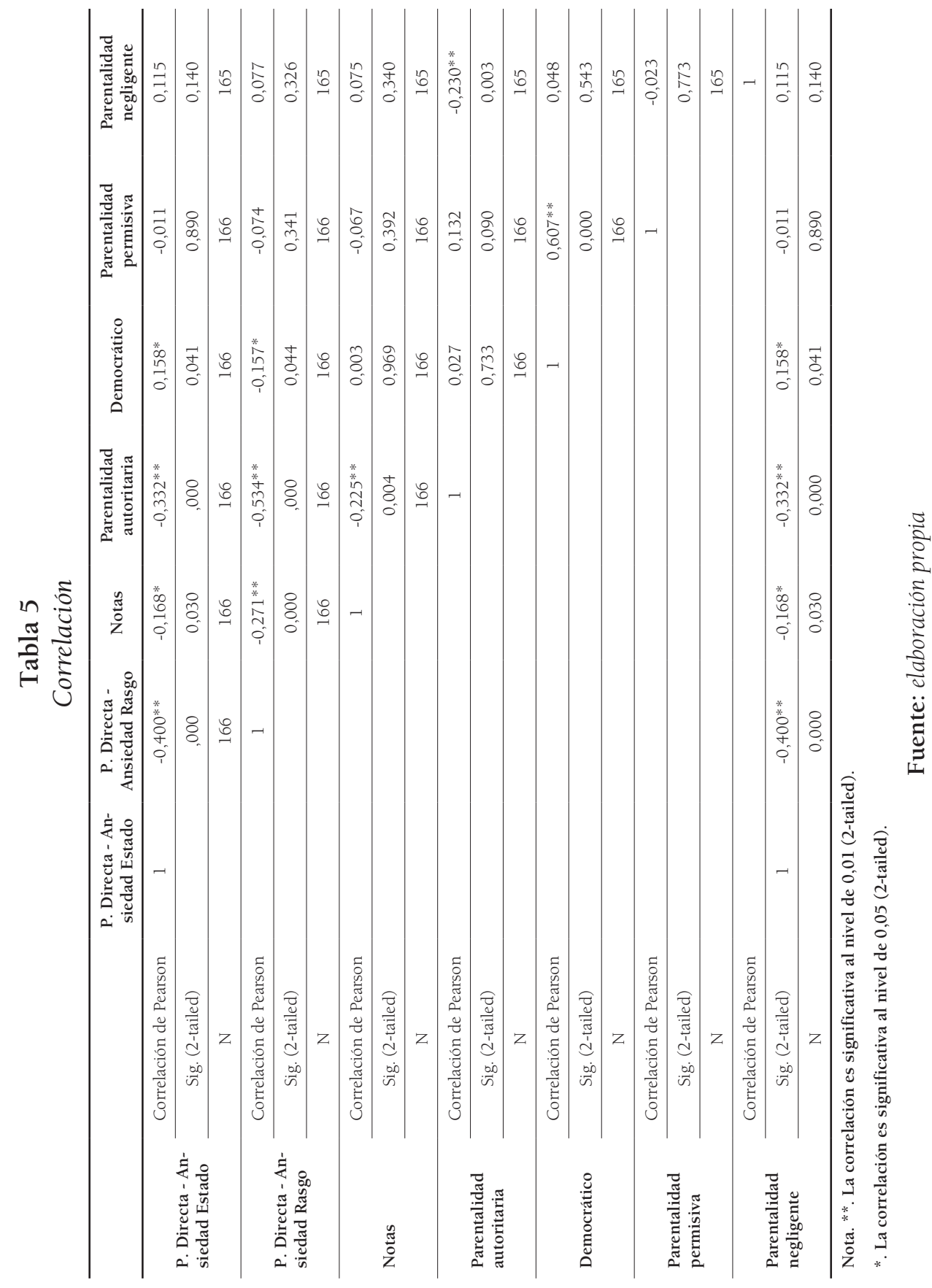




\section{Discusión}

La finalidad del presente estudio fue determinar si existe relación entre las prácticas parentales, la sintomatología ansiosa y el rendimiento académico de los estudiantes de secundaria de la Institución Educativa San Juan Bosco de San Pedro - Sucre. Los antecedentes que se encontraron en relación a las variables de estudio son en su gran mayoría internacionales, seguido de los nacionales, y por último, con menor cifra de resultados, los locales.

De acuerdo con los resultados obtenidos, y dado que se están evaluando los estilos parentales implicados en la muestra poblacional ya mencionada, se hace necesario remitirnos a los postulados de Bumrind (1967) quien afirma: los adolescentes que han sido educados por padres autoritarios tienen una mayor tendencia a ser retraídos en la parte social, además de tener dificultades para las relaciones interpersonales y la confianza en los demás, esto acompañado de conductas difíciles de manejar. Mientras tanto, en esta investigación los estudiantes formados bajo el estilo autoritario no presentan síntomas relacionados a la ansiedad.

Siguiendo con los planteamientos de Bumrind (1997) Los adolescentes que están bajo un estilo parental permisivo tienen más posibilidades de presentar conductas impulsivas, con bajo autocontrol, y al mismo tiempo, con baja tolerancia a la frustración, lo que no les permitirá tener una correcta adaptación a su medio.

Por otra parte, los hijos de padres negligentes tendrán mayor posibilidad de presentar conductas disruptivas y/o delictivas. Por último, los adolescentes que han sido criados por padres democráticos tienden a mostrar conductas más positivas, son más independientes y autosuficientes, demuestran una alta autoestima y respeto por los demás. Cabe acotar que, en el presente estudio los hijos con padres democráticos presentan mayor índice de ansiedad estado, caracterizado de manera funcional.

Sirviendo de fundamento a los hallazgos de este estudio, traemos a colación diversas investigaciones han descrito la existencia de una fuerte relación entre el comportamiento parental durante la crianza y comportamientos típicos de ansiedad, depresión, baja autoestima y estrés en adolescentes (Musitu, Estevez y Herrero, 2005; Sánchez y Postigo, 
2012; Linares y Fernández, 2015). En línea con estos hallazgos, en nuestro estudio, efectivamente, la ansiedad guarda una relación con los estilos parentales, destacando una correlación positiva con el estilo democrático, pero, una correlación negativa con el estilo autoritario.

Igualmente, Fajardo, Maestre, Felipe, León y Polo (2017), encontraron que el desempeño de los adolescentes en el colegio está influenciado por la formación académica, la clase social de sus padres y el apoyo brindado a sus hijos. Sin embrago, la relación en nuestro estudio fue vista desde la perspectiva de tipo de crianza, sin tener en cuenta el estrato social de los participantes. También en Gómez, Casas y Ortega-Ruiz (2016), se halló relación positiva entre la ansiedad y el comportamiento del padre y de la madre.

De la Torre, Casanova, García, Carpio y Cerezo (2011) encontraron que el estilo de crianza más prevalente era el autoritario (con 26,1\%) y el menos prevalente fue el negligente (con 23,8\%). A diferencia de nuestra investigación, el estilo de crianza que más prevaleció fue la Autoritativa, con un $39,2 \%$ y la menos relevante fue el estilo de crianza permisivo, con un 10,2\%. Más tarde, en 2105, estos autores descubrieron que los estudiantes con cuyos padres con estilos autoritarios y permisivos tienen mejor rendimiento, mientras que los estudiantes con padres con estilos autoritarios y negligentes tienen bajos rendimiento académico.

Contreras et al. (2005), descubrieron que la autoeficacia y la ansiedad son significativas en el rendimiento académico, por su parte, Moreno y Chauta (2012) enfatiza en que el nivel de disfunción familiar determina el grado de rendimiento académico, al igual que en esta investigación. No obstante, el presente análisis se hizo orientado al estilo parental más que al grado de funcionalidad familiar. Por lo contario, Díaz, Arrieta y Gonzalez (2014) descubrieron que los problemas académicos observados en estudiantes están más que todo ligados a falta de organización, problemas económicos, y por último, los problemas familiares. Cabe destacar que ese estudio fue realizado en estudiantes universitarios.

Es importante resaltar que en el presente estudio solo se tuvo en cuenta la sintomatología ansiosa, por lo que sería conveniente estudiar a futuro la sintomatología depresiva u otras variables que se enmarquen dentro del espectro de la psicología, por otra parte, el rendimiento académico debería 
ser medido, además de las notas académicas, mediante una prueba de inteligencia que exprese las habilidades concretas de las estudiantes.

\section{Conclusiones}

En la presente investigación se estudiaron tres variables: prácticas parentales, sintomatología ansiosa y rendimiento académico. Teniendo en cuenta el análisis de los resultados se determinaron las siguientes correlaciones: la ansiedad estado y la ansiedad rasgo correlacionaron negativamente con la parentalidad autoritaria. La ansiedad estado se correlaciona positivamente con el estilo parental democrático. Del mismo modo, el estilo parental democrático se correlaciona de forma negativa con la ansiedad rasgo. Por consiguiente, las notas guardan relación con la parentalidad autoritaria, es decir, que a mayor estilo parental autoritario, los estudiantes obtienen mejores notas, pero son menos ansiosos. También existe correlación con los estudiantes que presentan un bajo rendimiento académico y que se encuentran bajo el estilo de crianza negligente, asimismo, estos sujetos presentan un nivel de ansiedad elevado en la categoría rasgo, de acuerdo con lo evaluado por la prueba STAI.

Después de llevar a cabo el presente estudio y analizar todos los datos relacionados, se pudo corroborar el supuesto implícito planteado en la investigación, pues existe una relación significativa entre las variables: prácticas parentales, sintomatología ansiosa y rendimiento académico, estudiadas en la muestra poblacional.

Existió una correlación entre la ansiedad estados — descrita como una condición emocional transitoria- con el estilo parental autoritario, así como, con el estilo parental democrático, siendo este último el más empleado por lo cuidadores en la educación de sus hijos.

El estilo parental negligente influyó de forma desfavorable en las notas obtenidas por los estudiantes, considerándose este estilo el segundo más implementado en la crianza de los adolescentes, en tanto que representa $30,7 \%$ de la muestra poblacional. 


\section{Referencias}

Baumrind, D. (1967). Child Care Practices Anteceding Three Patterns. Of Preschool Behavior. Selection 22, Recuperado de http://homepages. utoledo.edu/mcaruso/honors-lifespan/baumrind.PDF.

Baumrind, D. (1966). effects of authoritative parental control on child behavior. Of Preschool Behavior. Selection. Recuperado de http:// persweb.wabash.edu/facstaff/hortonr/articles\%20for\%20class/ baumrind.pdf.

Contreras, F., Espinosa, J., Esguerra, G., Haikal, A., Polanía, A., y Rodríguez, A. (2005). Autoeficacia, ansiedad y rendimiento académico en adolescentes. Diversitas: Perspectivas en Psicología, 1(2), 183194. Recuperado de http://www.scielo.org.co/scielo.php?script=sci_arttext\&pid=S1794-99982005000200007\&lng=en\&tlng=.

De la Torre, M., Casanova, P., García, M., Carpio, M., \& Cerezo, M. (2011). Estilos educativos paternos y estrés en estudiantes de educación secundaria obligatoria. Psicología Conductual, 19(3), 577-590. Recuperado de https://search-proquest-com.ezproxy.cecar.edu.co:2443/ docview/927580532?accountid=34487.

Díaz, S., Arrieta k. \& Gonzalez, F. (2014). Estres academico y funcionalidad familiar en estudiantes de odontologia. Salud Uninorte, 30(2) Recuperado de https://search-proquest-com.ezproxy.cecar.edu.co:2443/ docview/1622345469? accountid=34487.

Espitia, L. y Montes, M. (2009). Influencia de la familia en el proceso educativo de los menores del barrio costa azul de Sincelejo (Colombia), scielo. Recuperado de http://www.scielo.org.co/pdf/indes/v17nl/ v17nla04.pdf.

Fajardo B, F, Maestre C. M., Felipe C. E., León B. B. \& Polo R. M. (2017). Análisis del rendimiento académico de los alumnos de Educación Secundaria Obligatoria según las variables familiares. Educación XX1, 20(1), 209-232, doi: 10.5944/educXX1.14475.

Gómez O., Casas, C., \& Ortega-Ruiz, R. (2016). Ansiedad social en la adolescencia: factores psicoevolutivos y de contexto familiar. Psicología Conductual, 24(1), 29-49. Recuperado de https://search-proquestcom.ezproxy.cecar.edu.co:2443/docview/1784859107?accoun$\operatorname{tid}=34487$. 
Hernández, R., Fernández, C., \& Baptista, M. (2010). Metodología de la investigación. Quinta Edición. México df: mcgraw-hill/interamericana editores, s.a de c.v.

Linares, M. \& Fernández, M. (2015). Las prácticas educativas paternas y la agresividad premeditada e impulsiva de los hijos adolescentes. Psicología Conductual, 23(1), 161-179. Recuperado de https://search-proquest-com.ezproxy.cecar.edu.co:2443/docview/1674473756? accountid=34487.

Lipps, G., Lowe, G., Gibson, R., Halliday, S., Morris, A., Clarke, N., y Wilson R. (2012). Child and Adolescent Psychiatry and Mental Health. Psiquiatría Infantil y Adolescente y Salud Mental. Recuperado de https://doi.org/10.1186/1753-2000-6-31.

Moreno, J., \& Chauta, L. (2012). Funcionalidad familiar, conductas externalizadas y rendimiento académico en un grupo de adolescentes de la ciudad de bogotá. Psychologia : Avances De La Disciplina, 6(1), 155-166. Recuperado de https://search-proquest com.ezproxy.cecar.edu.co:2443/docview/1793558826 accountid=34487.

Musitu Ochoa, G., Estévez López, E., Herrero Olaizola, J. (2005). El rol de la comunicación familiar y del ajuste escolar en la salud mental del adolescente. Salud Mental, 28() 81-89. Recuperado de http://4www.redalyc.org/articulo.oa?id=58242809.

Ospina, F., Hinestrosa M., Paredes, M., Guzmán, Y. y Granados, C. (2011). Síntomas de ansiedad y depresión en adolescentes escolarizados de 10 a 17 años en Chía, Colombia. Revista de Salud Pública, 13(6), 908-920. https://dx.doi.org/10.1590/S0124-00642011000600004.

OCDE (2016). Estudiantes de bajo rendimiento, por qué se quedan atrás y cómo ayudarles a tener éxito. Recuperado de http://www.oecd.org/ pisa/keyfindings/PISA-2012-Estudiantes-de-bajo-rendimiento.pdf.

Organización para la cooperación y el desarrollo económico (2016). PISA 2015 Resultados Clave. Recuperado de https://www.oecd.org/pisa/ pisa-2015-results-in-focus-ESP.pdf.

Programa para la Evaluación Internacional de Alumnos, PISA (2012). Estudiantes de bajo rendimiento por qué se quedan atrás y cómo ayudarles a tener éxito. Recuperado de http://www.oecd.org/pisa/keyfindings/PISA-2012-Estudiantes-de-bajo-rendimiento.pdf. 
Prácticas parentales, sintomatología ansiosa y rendimiento académico en estudiantes adolescentes, San Pedro - Sucre

Sánchez, M., y Postigo, J. (2012). Inteligencia emocional y clima familiar. Psicología Conductual, Recuperado de https://search-proquestcom.ezproxy.cecar.edu.co:2443/docview/1010354249?accoun$\mathrm{tid}=34487$.

Tonconi, J. (2010). Factores que influyen en el rendimiento académico y la deserción de los estudiantes de la facultad de ingeniería económica de la una-puno, periodo 2009. 2(1)//www.eumed.net/rev/ced/ index.htm. 